\title{
Microbial transformation of citral by Penicillium sp.
}

\author{
Akbar Esmaeili ${ }^{\bowtie}$ and Afsaneh Tavassoli \\ Department of Chemical Engineering, North Tehran Branch, Islamic Azad University, Tehran, Iran
}

Thymol is present in the essential oils from herbs and spices, such as thyme. It is produced by these plant species as a chemical defense against phytopathogenic microorganisms. Therefore, this compound has attracted great attention in food industry, i.e., it has been used as a natural preservative in foods such as cheese to prevent fungal growth. Previous studies concerning the biotransformation of nerol by Penicillium sp. and microbial transformation of citral by sporulated surface cultures method (SSCM) of Penicillium digitatum have been reported. The objective of this research was to study the pathway involved during biotransformation of citral by Penicillium sp. using two methods. The culture preparation was done using different microbial methods and incubation periods to obtain Penicillium for citral biotransformation. The biotransformation products were identified by gas chromatography (GC) and gas chromatography/mass spectroscopy (GC/MS). A comparison of the two methods showed that SSCM was more effective, its major products were thymol $(21.5 \%)$, geranial $(18.6 \%)$ and nerol (13.7\%). LM produced only one compound - thymol with a low efficiency.

Keywords: biotransformation, bioconversion, Penicillium sp., fungi, thymol, geranial

Received: 24 November, 2009; revised: 14 May, 2010; accepted: 08 July, 2010; available on-line: 14 September, 2010

\section{INTRODUCTION}

Biotransformation by cultured plant cells is an important method to convert cheap and plentiful organic compounds into more useful ones, according to the ability of plant cell cultures specifically to produce secondary metabolites (Suga \& Hirata, 1990; Shimoda, 2006). The reactions involved in the biotransformation of organic compounds by cultured plant cells include oxidation, reduction, hydroxylation, esterification, methylation, isomerization, hydrolysis, and glycosylation (Suga \& Hirata, 1990; Ishihara et al., 2003; Shimoda, 2006).

In the course of work related to bioconversion of monoterpene aldehyde by fungi, microbial transformation of citral by Penicillium sp. with the sporulated surface cultures method (SSCM) and liquid method (LM) approaches was investigated. The bioconversion of geranyl and neryl acetate ((Z)-2,6-octadien-1-ol-3,7-dimethyl acetate) by Aspergillus niger has been described (Madyastha et al., 1988a; 1988b). The main reaction found was hydrolysis of terpene acetates to the alcohols, followed by further $\omega$-hydroxylation to the respective 8-hydroxy derivatives. The degradation of geraniol by $A$. niger has also been examined by a Japanese group (Goto, 1967).
In 1964, use of edgeraniol was converted to linalool and partially oxidized to citral using the SSCM procedure (Wood, 1969). Microbial transformation of geraniol and nerol by SSCM of five $A$. niger strains and three Penicillium strains were compared with that using of submerged LM (Demyttenaere et al., 2000). It has been mentioned by several workers that different fungi can bioconvert citral (Massada et al., 1976; Ramaswami et al., 1988; Larroche et al., 1989; Adams, 1995; Demyttenaere et al., 1998).

In previous studies we done biotransformation of menthol by Mucor ramannianus, Penicillium sp. and $A$. niger. Using SSCM menthol was converted to oxygenated compounds. We found that those microorganisms are able to perform oxidations, reductions, hydrolytic reactions, dehydrations and formation of $\mathrm{C}-\mathrm{C}$ bonds and several degradation reactions (Scharf et al., 1986; Jansseens et al., 1991; Esmaeili et al., 2009a; 2009b).

In this article, microbial transformation of pure terpene aldehyde citral is carried out by SSCM, and the pathways involved in this microbial transformation are investigated. Microbial transformation of citral by Penicillium sp. SSCM grown on $50 \mathrm{ml}$ medium in conical flasks was monitored over three weeks. The suspension was extracted with diethyl ether three times and directly analyzed by GC and GC/MS.

\section{MATERIAL AND METHODS}

Microorganisms and cultivation. The Penicillium sp. strain was isolated from a soil sample collected in April 2008 in the province of Tehran, Iran. The culture was cultivated and conserved on Malt Extract Agar (MEA) (malt extract, $20 \mathrm{~g}$; peptone, $1 \mathrm{~g}$; dextrose, $20 \mathrm{~g}$; agar, $20 \mathrm{~g}$; distilled water, $1 \mathrm{l}$ ) for 10 days at $28^{\circ} \mathrm{C} \pm 2{ }^{\circ} \mathrm{C}$.

Experiments with SSCM. Spores were recovered from 3-week-old surface cultures of Penicillium sp. grown in Petri dishes on SDA (Merck). Spore suspension was prepared by adding $10 \mathrm{ml}$ of $0.2 \%$ sterile Tween 80 solution in distilled water. A total of $50 \mathrm{ml}$ of the spore suspension with $5 \times 10^{8}$ spore $/ \mathrm{ml}$ was obtained, which was shaken in a $250 \mathrm{ml}$ conical flask.

To this spore suspension $1 \mathrm{ml}$ of a solution of $5 \%$ citral in absolute ethanol was added, and the suspension was placed on a shaker at $180 \mathrm{rpm}$. After 5, 10 and 321 weekdays this suspension was extracted with $3 \times 50 \mathrm{ml}$ diethyl ether, and the products were directly analyzed by GC and GC/MS.

Experiments with LM. A $500 \mathrm{ml}$ Erlenmeyer flask containing $100 \mathrm{ml}$ medium (malt extract, $10 \mathrm{~g}$; pep-

e-mail: akbaresmaeili@yahoo.com

Abbreviations: GC, gas chromatography; GC/MS, gas chromatography/mass spectroscopy; LM, liquid method; $t_{\mathrm{R}^{\prime}}$ SSCM, sporulated surface cultures method 


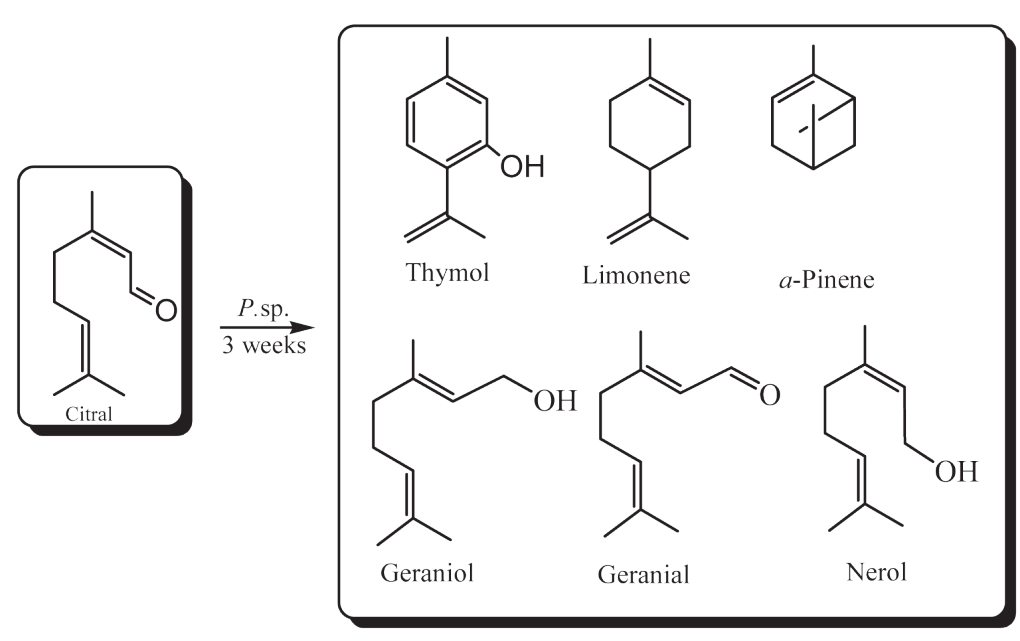

Figure 1. Identified products of bioconversion of citral by Penicillium sp. thymol (retention time $17.17 \mathrm{~min}$ ), geranial (retention time $17.21 \mathrm{~min}$ ), nerol (retention time $16.41 \mathrm{~min}$ ), geraniol (retention time $16.61 \mathrm{~min}$ ), $\alpha$-pinene (retention time $9.89 \mathrm{~min}$ ), and limonene (retention time 12.18 min), i.e., the six main peaks, are given below:

Thymol: $150\left[\mathrm{M}^{+}\right]$: 135(100), 150(30), 115(17), 91(16), 136(10), 77(10), 65(7), 121(5).

Geranial: $152\left[\mathrm{M}^{+}\right]$: 41(100), 69(62), 84(15), 53(10), 83(7), 94(7), 67(6), 55(5).

Geraniol: $154\left[\mathrm{M}^{+}\right]$: 41(100), 69(75), 81(15), 53(10), 93(7), 123(7), 111(6), 139(5).

$\alpha$-Pinene: $136\left[\mathrm{M}^{+}\right]$: 93(100), 91(43), 92(39), 77(29), 79(24), 105(17), 121(12), 139(5).

Limonene: $\quad 136\left[\mathrm{M}^{+}\right]$: 68(100), 67(86), 93(78), 79(43), 39(36), 53(34), 107(30), 121(30).

tone, $1 \mathrm{~g}$; dextrose, $20 \mathrm{~g}$; agar, $20 \mathrm{~g}$; distilled water, 1 l, $\mathrm{pH}$ 5.6) was inoculated with a $5 \mathrm{ml}$ suspension of Penicillium sp. $\left(5 \times 10^{8}\right.$ spore per $\left.\mathrm{ml}\right)$ and incubated at $28^{\circ} \mathrm{C} \pm 2{ }^{\circ} \mathrm{C}$ for 4 days on a rotary shaker operating at $100 \mathrm{rpm}$. After full growth of the microorganism, solution of citral $(100 \mu \mathrm{g})$ in ethanol $(0.2 \mathrm{ml})$ was added under sterile conditions to the culture. The incubation was then continued for a further 10 days at $28^{\circ} \mathrm{C} \pm 2{ }^{\circ} \mathrm{C}$. After the completion of the incubation, the culture was extracted with diethyl ether $(3 \times 100$ $\mathrm{ml})$.

Analysis of samples with GC/MS. GC analysis was performed on a Shimadzu $15 \mathrm{~A}$ gas chromatograph equipped with a DB-5 capillary column (50 $\mathrm{m} \times 0.2 \mathrm{~mm}$, film thickness $0.32 \mu \mathrm{m})$. Split/non-split injector and flame ionization detector were heated at $250^{\circ} \mathrm{C}$. Nitrogen was used as carrier gas $(1 \mathrm{ml} / \mathrm{min})$. The oven temperature was kept at $60^{\circ} \mathrm{C}$ for $3 \mathrm{~min}$ and then heated to $220^{\circ} \mathrm{C}$ with $5^{\circ} \mathrm{C} / \mathrm{min}$ rate and kept constant at $220^{\circ} \mathrm{C}$ for $5 \mathrm{~min}$. Relative content of compounds was calculated from peak area using a Shimadzu C-R4A chromatopac integrator without correction.

GC/MS analysis was performed using a Hewlett-Packard 5973 device with an HP-5MS column $(30 \mathrm{~m} \times 0.25$ $\mathrm{mm}$, film thickness $0.25 \mu \mathrm{m})$. The oven temperature was kept at $60^{\circ} \mathrm{C}$ for $3 \mathrm{~min}$ and programmed to $220^{\circ} \mathrm{C}$ at a rate of $5^{\circ} \mathrm{C} / \mathrm{min}$ and kept constant at $220^{\circ} \mathrm{C}$ for $5 \mathrm{~min}$. The flow rate of helium a carrier gas was $1 \mathrm{ml} / \mathrm{min}$. MS were taken at $70 \mathrm{eV}$. Identification of the constituents of the oil was made by comparison of their mass spectra and retention indices (RRI) with those given in the literature and are authentic samples (Frank et al., 1977; Chen et al., 1982; Larroche et al., 1989). Mass spectra of

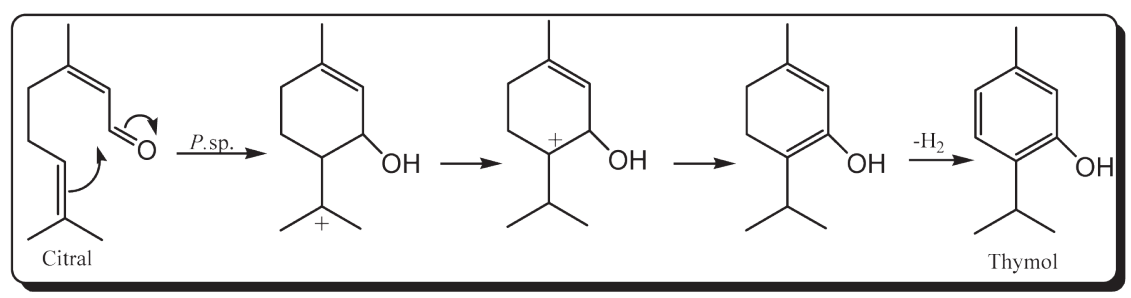

Figure 2. Suggested pathway of thymol formation from citral
Nerol: 154[M+]: 41(100), 69(45), 81(15), 53(10), 93(10), 121(7), 111(3), 139(2).

\section{RESULTS}

The microbial transformation of volatile monoterpenoids by fungal SSCM and LM were examined. The results suggested that LM is not suitable for small amount of sample, because this method can only produce one product (thymol) with a very low yield. In the SSCM method we identified six components representing $67.4 \%$ in the bioconversion. The main products obtained in the bioconversion of citral were thymol $(21.5 \%)$, geranial $(18.6 \%)$, nerol $(13.7 \%)$, geraniol $(6.8 \%), \alpha$-pinene $(3.7 \%)$ and limonene $(3.1 \%)$ (Fig. 1).

From the data given in Fig. 1 it can be concluded that citral was mostly converted to thymol, geranial and nerol. This pathway of citral conversion involved formation of a cyclic compound with a single double bond and finally gave an aromatic compound (thymol) (Fig. 2).

Production of geranial $(18.6 \%)$ and nerol $(13.6 \%)$ suggests that two isomers of citral were converted $-E$ and $Z$, respectively. Geranial is more stable than nerol because in the E-isomer the conjugatated double bond is in the trans configuration with the carbonyl bond (Fig. 3).

The time course of the bioconversion was as follows. After 5 days the products obtained in the bioconversion of citral were geraniol $(12.22 \%)$, geraniol formate $(2.215 \%)$ and geranial $(2.434 \%)$.

After 10 days the mass spectra showed 9-octadecenoic acid, hexadecanoic acid and thiazole. These compounds have not been observed in natural products (Adams, 1995).

After 21 days (3 weeks) the main products obtained in the bioconversion of citral were thymol $(21.5 \%)$, geranial $(18.6 \%)$, nerol $(13.7 \%)$, geraniol $(6.8 \%), \alpha$-pinene $(3.7 \%)$ and limonene $(3.1 \%)$. The highest yield of citral bioconversion was obtained after 18 days $(67.4 \%)$. 


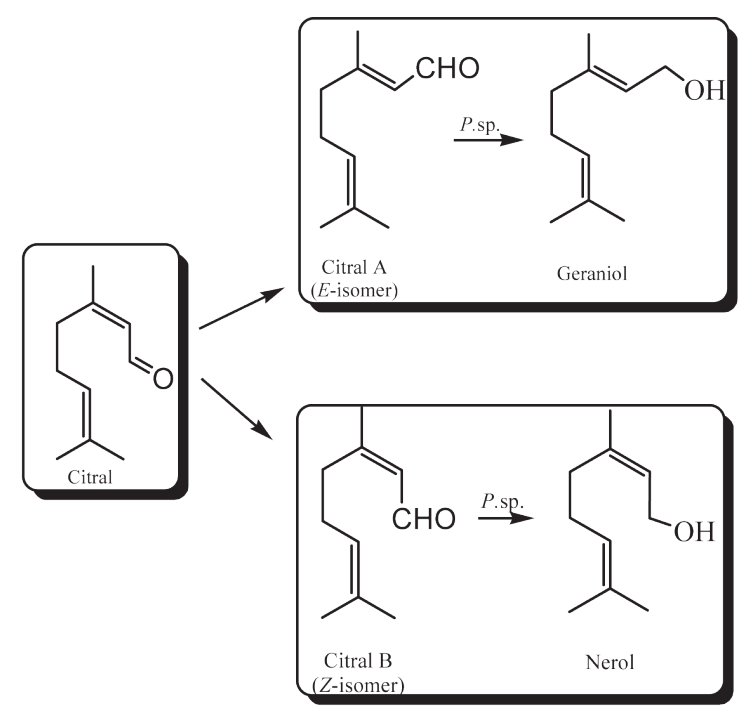

Figure 3. Possible pathway of bioconversion of two isomers of citral to geraniol and nerol

\section{DISCUSSION}

In previous studies of bioconversion of citral and nerol by spores of Penicillium digitatum, these were transformed into 6-methylhept-5-en-2-one (Demyttenaere et al., 1998). Microbial transformation of geraniol, nerol, and citral by $A$. niger produced linalool and $\alpha$-terpineol. Bioconversion of nerol with Penicillium chrysogenum mainly yielded $\alpha$-terpineol and some unidentified compounds. With Penicillium rugulosum the major bioconversion product from nerol and citral was linalool (Demyttenaere et al., 2000). Microbial transformation of menthol by SSCM of $A$. niger and Penicillium sp. produced terpineol and terpineol limonene, $p$-cymene, $\gamma$-terpinene, respectively (Esmaeili et al., 2009c). The two main products of microbial transformation of citral were similar to those obtained in the mentioned works. The main bioconversion products of (-)-menthol by sporulated surface cultures Mucor ramannianus were trans- $p$-menthan-8-ol, trans-menth2-en-1-ol, sabinane, $p$-menthane-3,8-diol, isomenthol, and 1,8-cineole (Esmaeili et al., 2009). The main biotransformation products obtained from menthol by surface grown Penicillium sp. were $\alpha$-pinene $(18.0 \%)$, terpineol $(10.6 \%)$, menthene $(5.8 \%)$, sabinene $(3.9 \%)$, 1,8-cineole $(6.4 \%)$, and limonene $(3.2 \%)$ (Esmaeili et al., 2009c). The cited results suggest that microbial transformation of monoterpenes with Penicillium and Aspergillus involved an oxidation reaction and resulted in a more stable product. But bioconversion using SSCM and LM of Penicillium showed that it was possible to obtain similar product with high yield and selectivity. Thymol can be produced by removal of hydrogen and rearrangement of citral. Thymol is present in the essential oils from herbs and spices. It is produced by these plant species as a chemical defense against phytopathogenic microorganisms. Therefore, this compound has attracted great attention in food industry. It has been used as a natural preservatives in foods such as cheese to prevent fungal growth.

\section{CONCLUSIONS}

1. In this research, microbial transformation of citral by Penicillium sp. is studied using two types of culture.

2. The major components of the microbial transformation of citral using the SSCM approach appeared to be thymol $(21.5 \%)$, geranial $(18.6 \%)$ and nerol $(13.7 \%)$ with an overall yield of $67.4 \%$.

3. The results suggested that LM is not suitable for small amount of sample because this method can produce only one product (thymol) with very poor yield $(<0.1 \%)$.

4. A proposed pathway of citral bioconversion to thymol is presented in Fig. 2.

5. SSCM can keep its activity over prolonged period of 3 weeks. Spores can also be entrapped, e.g., in Ca-alginate, and used for continuous production of aroma compounds, a system well known for the bioconversion of fatty acids to methyl ketones. Finally, many different terpenoid compounds can be tested for bioconversion reactions with the same batch of fungal spores.

\section{Acknowledgements}

The authors wish to thank Dr. Parviz Abromand, Department of Chemistry, Science \& Research Campus, Islamic Azad University (Tehran, Iran) for assiatance with the use of GC/MS.

\section{REFERENCES}

Adams R (1995) Identification of Essential Oil Components by Gas Chromatography/Mass Spectroscopy. Allured Publ. Corp., Carol Stream. IL,USA.

Chen FC, Chenm CF, Wei RD (1982) Acute toxicity of PR Toxin, a mycotoxin from Penicillium roqueforti. Toxicol 20: 431-433.

Demyttenaere JCR, Carme Herrera M, De Kimpe N (2000) Biotransformation of geraniol, nerol and citral by sporulated surface cultures of Aspergillus niger and Penicillium sp. Phytochemistry 55: 363-373.

Demyttenaere JCR, De Pooter HL (1998) Biotransformation of citral and nerol by spores of Penicillium digitatum. Flav Frag J 13: 10291036.

Esmaeili A, Sharafian S, Safaiyan S, Rezazadeh S, Rustaiyan A (2009a) Biotransformation of one monoterpene by sporulated surface cultures of Aspergillus niger and Penicillium sp. Nat Product Res 23: 1058 1061.

Esmaeili A, Saad N, Safaiyan S, Rustaiyan A (2009b) Biotransformation of (-)-menthol by spores of Mucor ramannianus and study of the pathways involved. Herba Polonica 55: 51-59.

Esmaeili A, Hoseiny Zarea A, Sharafian S Safaiyan S, Rustaiyan A (2009c) Biotransformation of menthol by sporulated surface cultures of Penicillium sp. and study of the pathways involved. Herba Polonica 55: 110-116.

Ishihara K, Hamada H, Hirata T, Nakajima N (2003) Biotransformation using plant cultured cells. J Mol Catalysit B: En₹ 23: 145-170.

Frank RK, Orth R, Ivankovic S, Kuhlmann M, Schmähl D (1977) Investigations on carcinogenic effects of Penicillium caseicolum and $P$. roqueforti in rats. Experentia 33: 515-516.

Goto K (1967) Biochemical degradation of geraniol by Aspergillus niger. Obibiro Chikusandaiagaku Gakujutsu Kenkyu Hokoku 4: 489-496; Chemical Abstracts 68;19904y,1968.

Ishihara K, Hamada H, Hirata T, Nakajima N (2003) Biotransformation using plant cultured cells. J Mol Catalysit B: Enz 23: 145-170.

Janssens L, De Pooter HL, Schamp NM, Vandamme EJ (1992) Production of flavours by microorganism. Proc Biochem 27: 195-198.

Larroche C, Arpah M, Gros JB (1989) Methylketone by Caalginate/Eudrasit RL entrapped spores of Penicillium roqueforti. Ensyme Microbial Technol 11: 106-112.

Larroche C, Gros JB (1989) Batch and continuous heptanone production Caalginate/Eudrasit RL entrapped spores of Penicillium roqueforti application to aroma production. Biotechnol Bioenginer 34: 30-38.

Madyastha KM, Krishna Murthy NSR (1988a) Transformation of acetates of citronellol, geraniol and linalool by Aspergillus niger: regiospe- 
cific hydroxylation of citronellol by a cell-free system. Appl Microb Biotechnol 28: 324-329.

Madyastha KM, Krishna Murthy NSR (1988b) Regiospecific hydroxylation of acyclic monoterpene alcohols by Aspergillus niger. Tetrah Lett 29: 579-580.

Massada Y (1976) In Analysis of essential oil by gas chromatography and mass spectrometry. Wiley, New York.

Scharpf LG Jr, Seitz EW, Morris JA, Farbood MI (1986) Generation of flavour and odour compounds through fermentation processes. In Biogeneration of Aromas. Parliment TH, Croteau R, eds, 317, pp 323-346. American Chemical Socity, Washington, DC, ACS Symposium Series.
Shimoda K, Kondo Y, Nishida T, Hamada H, Nakajima N, Hamada $\mathrm{H}$ (2006) Biotransformation of thymol, carvacrol, and eugenol by cultured cells of Eucalyptus perriniana. Phytochemistry 67: 2256-2261.

Suga T, Hirata T (1990) Biotransformation of exogenous substrates by plant cell cultures. Phytochemistry 29: 2393-2406.

Ramaswami SK, Briscese P, Gargiullo R, Vonngeldern T (1988) In Flavours and Fragrances. A World Perspective. Lawrence BM, Mookerjee BD, Willis BJ, eds. Elsevier: Amsterdam 951.

Wood JB (1969) Microbial fermentation of lower terpenoids. Process Biochem 2: 50-52. 